\title{
Selection of Model Parameters for Off-Line Parameter Estimation
}

\author{
Rujun Li, Michael A. Henson, and Michael J. Kurtz
}

\begin{abstract}
Mechanistic dynamic models often contain unknown parameters whose values are difficult to determine even with highly specialized laboratory experiments. A practical approach is to estimate such parameters from available process data. Typically only a subset of the parameters can be estimated due to restrictions imposed by the model structure, lack of measurements, and limited data. We present a simple parameter selection method which accounts for the first two factors independent of the data available for parameter estimation. The magnitude of each parameter effect on the measured variables is quantified by applying principal-component analysis to the steady-state parameter-output sensitivity matrix. The uniqueness of each parameter effect is determined by computing the minimum distance between the sensitivity vector of the particular parameter and the vector spaces spanned by sensitivity vectors of the parameters already selected for estimation. A recursive algorithm that provides a tradeoff between the magnitude and linear independence of parameter effects yields a ranking of the parameters according to their inherent ease of estimation. The parameter-selection procedure is applied to the problem of kinetic parameter estimation for an industrial model of a polymerization reactor. For this specific example, the proposed method yields superior estimation results than those obtained with a parameter-selection technique based on the Fisher information matrix (FIM).
\end{abstract}

Index Terms-Optimization, parameter estimation, polymer reactors.

\section{INTRODUCTION}

$\mathbf{N}$ ONLINEAR dynamic models derived from basic principles of physics and chemistry often contain parameters whose values cannot be accurately predicted from theory. Some parameter values are not available in the literature and specialized lab experiments aimed at determining these values often are infeasible. In this case, parameter values must be determined from process data. Systematic design of experiments to generate data well suited for estimating unknown parameters is an important research area [1], [2]. In chemical manufacturing plants, designed experiments often are impractical due to manufacturing constraints on product quality and throughput. Consequently, the development of parameter-estimation techniques which utilize readily available plant data is necessary.

Manuscript received April 1, 2002; revised May 9, 2003. Manuscript received in final form July 21, 2003. Recommended by Editor F. Doyle. This work was supported by the ExxonMobil Chemical Company.

$\mathrm{R}$. $\mathrm{Li}$ is with the Department of Chemical Engineering, Louisiana State University, Baton Rouge, LA 70803-7303 USA.

M. A. Henson is with the Department of Chemical Engineering, University of Massachusetts, Amherst, MA 01003-9303 USA (e-mail: henson@ecs.umass.edu).

M. J. Kurtz is with the ExxonMobil Chemical Company, Baton Rouge, LA 70805-3359 USA.

Digital Object Identifier 10.1109/TCST.2004.824799
The off-line parameter-estimation problem often is formulated as an optimization problem in which the unknown parameters are the decision variables and the least-squares difference between the measurements and the model predictions is minimized subject to constraints imposed by the model equations and known bounds on the parameters [3], [4]. Due to the model structure and possible lack of measurements, estimation of some parameters may be impossible regardless of the amount of data available. A nonlinear system is said to be structurally identifiable if each set of parameter values yields unique output trajectories [5]. Structurally unidentifiable parameters must be removed from the estimation problem because they have no effect on the measured outputs. However, structural identifiability only is a necessary condition for successful parameter estimation from limited data sets. Two additional problems are commonly encountered in practice.

1) A parameter has a very weak effect on the measured outputs. Successful estimation of such a weakly identifiable parameter is unlikely because its effect cannot be accurately quantified.

2) The effects of certain parameters on the measured outputs are nearly linearly dependent. Successful estimation of such parameter sets is unlikely because the individual parameter effects cannot be distinguished.

The presence of parameters with weak and/or nearly linearly dependent effects is manifested by the lack of a unique solution to the optimization problem for different initial parameter values. Estimation of such parameters can lead to significant degradation in the predictive capability of the model.

The development of an effective solution to the parameter selection problem requires establishing an acceptable tradeoff between the magnitude and linear independence of parameter effects. A parameter is said to be practically identifiable if a unique estimate can be obtained from different initial values using the available data [6]. Most methods to access practical identifiability are based on scalar measures derived from the Fisher information matrix (FIM) [5], [7], which can be computed from the parameter-output sensitivity coefficients. Consider a continuous-time, state-space model with discrete outputs

$$
\begin{aligned}
\frac{d x(t)}{d t} & =f[x(t), u(t), \theta] \\
y(k) & =h[x(k)]
\end{aligned}
$$

where: $t$ and $k$ denote continuous and discrete time, respectively, and $x, u, y$, and $\theta$ are the state, input, output, and parameter vectors, respectively. The parameter-output sensitivity 
coefficient $S_{i j}$ of the $i$ th output with respect to the $j$ th parameter is defined as [8]

$$
S_{i j}(k)=\frac{\partial y_{i}(k)}{\partial \theta_{j}} .
$$

Under the assumption that the measurements are corrupted by zero-mean white-Gaussian noise, the FIM $(F)$ can be computed as [9]

$$
F=\sum_{k=1}^{M} S^{T}(k) Q^{-1}(k) S(k)
$$

where $M$ is the total number of discrete samples, $S(k)=\left\{S_{i j}(k)\right\}$ is the sensitivity matrix, and $Q(k)$ is the measurement covariance matrix. The inverse of the FIM provides a lower bound on the parameter error covariance matrix [2].

Several scalar measures of the FIM have been proposed to select parameters best suited for estimation [10], [11]. Several investigators have proposed the use of multiple objectives to achieve better results. For example, a parameter selection procedure which utilizes both the FIM determinant and the FIM condition number has been applied to a standard model of the activated sludge process [12]. First the parameter-sensitivity matrix is computed to identify insensitive parameters that are eliminated from the subsequent analysis. Then the FIM is calculated for all possible permutations of the remaining $n$ parameters for subsets between 2 and $n$ parameters. For each subset size, all permutations are ranked according to the values obtained for FIM determinant and the FIM condition number. The user is required to heuristically analyze the two rankings to determine the "best" combination of parameters for each subset size. Finally the estimation results achievable with the candidate parameter sets are analyzed via simulation. In addition to being computationally inefficient, this combined FIM approach suffers from the heuristic nature of the parameter selection process.

In this paper, a simple method for selecting estimated parameters from a set of unknown parameters is presented. Principal component analysis (PCA) [13] is performed on a dimensionless version of the parameter-output sensitivity matrix. The overall steady-state effect of each parameter on the measured outputs is determined from the principal components and the associated eigenvalues computed from the sensitivity matrix. The uniqueness of each parameter effect is determined by computing the minimum distance between the sensitivity vector of the particular parameter and the vector spaces spanned by sensitivity vectors of the parameters already selected for estimation. The parameters are ranked recursively to achieve an acceptable tradeoff between the magnitude and linear independence of their effects. The proposed method is applied to the problem of kinetic parameter estimation for an industrial model of an ethylene-propylene-diene terpolymer reactor. Simulation tests are used to determine the number of parameters that can be determined uniquely from dynamic data.

The proposed method has been extended to periodic dynamics by considering the amplitude and mean value of each measurement as well as the oscillation period as output variables in the sensitivity calculations [14]. Instead of checking linear independence of parameter sets, a simple collinearity measure restricted to parameter pairs was used to identify linearly dependent effects. This modified-parameter selection method has been applied successfully to the selection of estimated parameters in a cell population balance equation model used to describe oscillatory dynamics in continuous yeast cultures.

\section{Chemical Process Example}

\section{A. EPDM Reactor Dynamic Model}

Ethylene-propylene (EPM) copolymers and ethylene-propylene-diene (EPDM) terpolymers are used in the manufacturing of automotive components, cable and wire, appliances, building and construction materials, and agricultural equipment [15]. Major US producers of EPM and EPDM include ExxonMobil Chemical, DuPont, Uniroyal Chemical, and DSM Copolymer. The annual domestic production of these polymers in 1995 was almost 400000 metric tons [16]. Product quality is determined primarily by the relative amounts of the ethylene, propylene, and diene monomers in the polymer and the Mooney viscosity (a commonly measured quantity related to the molecular weight). EPDM plants are used to manufacture a large number of distinct polymer grades that cover a wide range of product quality specifications.

EPDM typically is manufactured via a solution polymerization process which utilizes Ziegler-Natta catalyst technology. The core of the EPDM process is a continuous stirred tank reactor which is fed the three monomers, solvent (hexane or methyl chloride), catalysts (a transition metal halide catalyst and an aluminum alkyl co-catalyst), and chain-transfer agent (hydrogen). Stable reactor operation is essential to minimize off-specification product and to maximize production rates. Current industrial practice is to establish reactor operating conditions that are expected to maintain the product quality measures within their specifications. When statistical quality-control tests indicate the polymer is off-specification, manual adjustments are made to various feed flow rates in an attempt to achieve the target product properties. Because EPDM reactors are highly nonlinear and interacting, nonlinear model-based control strategies offer the potential for significantly improved performance.

A mechanistic-reactor model is based on an assumed-kinetic scheme that describes the major reactions that produce the polymer chains. Several EPDM kinetic models have been presented in the open literature [17]-[19]. In this paper, we utilize the kinetic model of Cozewith [17] shown in Table I. The basic reaction steps are: i) formation of an active catalyst species $\left(C_{2}\right)$ from the catalyst $\left(C_{1}\right)$ and co-catalyst $(A l)$; ii) deactivation of the catalyst with various poisons such as diene monomer $\left(M_{3}\right)$ to form dead catalyst species $(D)$; iii) initiation of a growing polymer chain by reaction of an active catalyst species with ethylene monomer $\left(M_{1}\right)$ or propylene monomer $\left(M_{2}\right)$; iv) propagation of a polymer chain by the addition of a monomer unit to the growing chain; v) termination of polymer-chain growth by the transformation of a growing chain to a dead chain; and vi) transfer of the active catalyst from a growing polymer chain to a new polymer chain by the action of various 
TABLE I

EPDM KINETIC MECHANISM

\begin{tabular}{|c|c|c|}
\hline Type & Reaction & Rate Constant \\
\hline 1. Catalyst activation & $C_{1} \rightarrow C_{2}$ & $k_{a}$ \\
\hline \multirow[t]{2}{*}{ 2. Catalyst deactivation } & $C_{1} \rightarrow D$ & $k_{x}$ \\
\hline & $C_{1}+M_{3} \rightarrow D$ & $k_{x 3}$ \\
\hline \multirow[t]{2}{*}{ 3. Chain initiation } & $C_{2}+M_{1} \rightarrow P_{100}$ & $k_{i 1}$ \\
\hline & $C_{2}+M_{2} \rightarrow Q_{010}$ & $k_{i 2}$ \\
\hline \multirow[t]{6}{*}{ 4. Chain propagation } & $P_{i j k}+M_{1} \rightarrow P_{(i+1) j k}$ & $k_{11}$ \\
\hline & $P_{i j k}+M_{2} \rightarrow Q_{i(j+1) k}$ & $k_{12}$ \\
\hline & $P_{i j k}+M_{3} \rightarrow R_{i j(k+1)}$ & $k_{13}$ \\
\hline & $Q_{i j k}+M_{1} \rightarrow P_{(i+1) j k}$ & $k_{21}$ \\
\hline & $Q_{i j k}+M_{2} \rightarrow Q_{i(j+1) k}$ & $k_{22}$ \\
\hline & $R_{i j k}+M_{1} \rightarrow P_{(i+1) j k}$ & $k_{31}$ \\
\hline \multicolumn{3}{|l|}{ 5. Chain termination } \\
\hline \multirow[t]{3}{*}{ spontaneous } & $P_{i j k} \rightarrow \overline{U_{i j k}}$ & $k_{t}$ \\
\hline & $Q_{i j k} \rightarrow V_{i j k}$ & $k_{t}$ \\
\hline & $R_{i j k} \rightarrow W_{i j k}$ & $k_{t}$ \\
\hline \multirow[t]{3}{*}{ with propylene } & $\bar{P}_{i j k}+M_{2} \rightarrow U_{i j k}$ & $k_{t 2}$ \\
\hline & $Q_{i j k}+M_{2} \rightarrow V_{i j k}$ & $k_{t 2}$ \\
\hline & $R_{i j k}+M_{2} \rightarrow W_{i j k}$ & $k_{t 2}$ \\
\hline \multirow[t]{3}{*}{ with diene } & $P_{i j k}+M_{3} \rightarrow U_{i j k}$ & $k_{t 3}$ \\
\hline & $Q_{i j k}+M_{3} \rightarrow V_{i j k}$ & $k_{t 3}$ \\
\hline & $R_{i j k}+M_{3} \rightarrow W_{i j k}$ & $k_{t 3}$ \\
\hline \multicolumn{3}{|l|}{ 6. Chain transfer } \\
\hline \multirow[t]{3}{*}{ with hydrogen } & $P_{i j k}+H_{2} \rightarrow U_{i j k}+C_{2}$ & $k_{t r 1}$ \\
\hline & $Q_{i j k}+H_{2} \rightarrow V_{i j k}+C_{2}$ & $k_{t r 1}$ \\
\hline & $R_{i j k}+H_{2} \rightarrow W_{i j k}+C_{2}$ & $k_{t r 1}$ \\
\hline \multirow[t]{3}{*}{ with cocatalyst } & $P_{i j k}+A l \rightarrow U_{i j k}+P_{100}$ & $k_{t r}$ \\
\hline & $Q_{i j k}+A l \rightarrow V_{i j k}+P_{100}$ & $k_{t r}$ \\
\hline & $R_{i j k}+A l \rightarrow W_{i j k}+P_{100}$ & $k_{t r}$ \\
\hline \multirow[t]{3}{*}{ with propylene } & $P_{i j k}+M_{2} \rightarrow U_{i j k}+Q_{010}$ & $k_{t r_{M 2}}$ \\
\hline & $Q_{i j k}+M_{2} \rightarrow V_{i j k}+Q_{010}$ & $k_{t r_{M 2}}$ \\
\hline & $R_{i j k}+M_{2} \rightarrow W_{i j k}+Q_{010}$ & $k_{t_{M 2}}$ \\
\hline
\end{tabular}

chain transfer agents such as hydrogen $\left(\mathrm{H}_{2}\right)$. The symbols $P_{i j k}$ and $U_{i j k}$ are used to represent ethylene-ended growing and dead chains, respectively, with a total number of $i$ ethylene units, $j$ propylene units, and $k$ diene units. Analogous notation is used for propylene chains $\left(Q_{i j k}, V_{i j k}\right)$ and diene chains $\left(R_{i j k}, W_{i j k}\right)$.

The dynamic reactor model consists of mass balances for each reactant combined with balances for various moments of the molecular weight distribution (MWD) that are derived from the kinetic scheme in Table I. An energy balance is not required because EPDM reactors are operated isothermally under normal conditions. The rate of each elementary reaction $j$ is characterized by a rate constant $k_{j}$. Defining $\theta$ to be a vector comprised of the rate constants chosen for estimation, the dynamic model equations have the following form:

$$
\frac{d x_{i}}{d t}=\frac{F}{V}\left(x_{i, f}-x_{i}\right)+r_{i}(x, \theta)
$$

where: $x_{i}$ is the $i$ th state variable which represents a reactant molar concentration or MWD moment, $x_{i f}$ is the concentration of the $i$ th state variable in the feed stream which is nonzero only for the reactants, $F$ is the feed volumetric flow rate, $V$ is the constant reactor volume, and $r_{i}$ is a reaction rate function that depends nonlinearly on the state variables and linearly on the parameters [19]. The complete model has 29-state variables representing seven reactant concentrations, four zeroth-order moments, 12 first-order moments and six-bulk second-order mo-
TABLE II

SCALED REACTION Rate CONSTANTS

\begin{tabular}{c|c|c|c}
\hline Parameter & Value & Parameter & Value \\
\hline$k_{a}$ & 0.3663 & $k_{21}$ & 0.6410 \\
\hline$k_{x}$ & 0 & $k_{22}$ & 3.0037 \\
\hline$k_{x_{2}}$ & 0 & $k_{31}$ & 1.2418 \\
\hline$k_{x_{3}}$ & 0.9890 & $k_{t}$ & 0.5604 \\
\hline$k_{i 1}$ & 1.8315 & $k_{t_{2}}$ & 0.7839 \\
\hline$k_{i 2}$ & 1.8315 & $k_{t_{3}}$ & 0 \\
\hline$k_{11}$ & 0.4212 & $k_{t r}$ & 0 \\
\hline$k_{12}$ & 0.6886 & $k_{t r_{1}}$ & 1.7582 \\
\hline$k_{13}$ & 0.4139 & $k_{t r_{2}}$ & 1 \\
\hline
\end{tabular}

ments. Detailed model equations are not provided here for the sake of brevity. The interested reader is referred to [19] for further details on the general model structure.

\section{B. Parameter Estimation}

Although EPDM reaction kinetics have been studied extensively, a complete and consistent set of reaction-rate constants is difficult to find in the open literature. A few parameter sets for EPM copolymers and EPDM terpolymers are tabulated in [17]. The available information usually is not sufficient to completely specify the 18-rate constants involved in the kinetic mechanism. This problem is exasperated by the fact that commercial plants are used to manufacture a wide range of polymer grades that differ with respect to the catalyst system, diene monomer type, and nominal operating conditions. The determination of complete parameter sets from the literature and specialized laboratory experiments is not feasible. An alternative approach is to estimate the kinetic parameters from readily available plant data.

To perform off-line parameter estimation, a nominal set of kinetic rate constants that serve as initial conditions in the optimization problem must be specified. We utilize the parameter values listed in Table II because they are the best available estimates for the particular polymer grade studied in this paper. Each parameter value has been scaled to have roughly the same order of magnitude. Accuracy of the parameter estimates is strongly dependent on the available plant measurements. We assume that the polymer-production rate $\left(P_{r}\right)$, the polymer contents of ethylene $\left(X_{M_{1}}\right)$ and diene $\left(X_{M_{3}}\right)$, and the weight-average molecular weight $\left(\bar{M}_{w}\right)$ are measured every six minutes without delay. Although not pursued in this study, the Mooney viscosity (a primary indicator of product quality) can be inferred from $\bar{M}_{w}$ [19].

The off-line parameter-estimation problem is formulated as the constrained nonlinear optimization problem $\min _{\theta} J$ where

$$
J=\frac{1}{M} \sum_{k=1}^{M} \sum_{i=1}^{m}\left[\frac{y_{i}(k)-y_{p, i}(k)}{y_{p, i}(k)}\right]^{2}
$$

$m$ is the vector of measurements and $y_{p, i}$ is the $i$ th plant measurement. The minimization is performed subject to equality constraints imposed by the model (1) and inequality constraints that ensure positive kinetic parameter estimates $(\theta \geq 0)$. We utilize a sequential-solution strategy in which optimization and model integration are performed separately [20]. The optimization problem (5) generally is nonconvex due to the nonlinear model equations [21]. We have found that gradient based opti- 
mization algorithms such as sequential-quadratic programming [22] and direct-search algorithms such as the simplex method [23] often converge to local minima. Better performance has been obtained with probabilistic-optimization codes such as the genetic algorithm [24] and simulated annealing (SA) [25]. Although convergence to the global minimum is not guaranteed, the SA code [26] used in this paper yields good results and is much easier to tune than genetic algorithm codes. The main shortcoming of the SA algorithm is its computational inefficiency.

Under the assumption that the nonlinear-dynamic model is structurally identifiable, perfect estimation of every unknown parameter is theoretically possible. In practice, good estimates cannot be obtained for some of the parameters due to lack of measurements and because the operating range is severely limited by manufacturing constraints. The presence of parameters with weak and/or almost linearly-dependant effects often results in convergence to different local minima from different initial guesses. Because mechanistic-nonlinear models invariably contain such parameters, only a subset of the unknown parameters should be selected for estimation.

\section{PARAmEter Selection for OfF-Line Estimation}

The proposed parameter-selection method is based on the parameter-output sensitivity matrix computed at a particular steady-state operating point. As such, the results obtained are local with respect to the operating point and the initial values of the unknown parameters. Successful application of the method requires that: i) the steady-state behavior is not strongly dependent on the operating point such that the results are extensible to other operating conditions and ii) the initial parameter values produce reasonable qualitative predictions such that order of magnitude variations are not required to achieve quantitative agreement with plant data. Many industrial processes including EPDM reactors satisfy these conditions due to infrequent changes in operating conditions and the availability of reasonable parameter values from previous modeling efforts. Possible extensions of the parameter-selection method for handling nonlinearities and dynamics are discussed in the concluding section.

\section{A. Magnitude of Parameter Effect}

A new measure for the overall steady-state effect of a model parameter on a set of measured-output variables is presented. The measure is derived from the dimensionless parameter-output sensitivity coefficients $\tilde{S}_{i j}$ defined as

$$
\tilde{S}_{i j}=\frac{\tilde{\theta}_{j}}{\tilde{y}_{i}} \frac{\partial \bar{y}_{i}}{\partial \theta_{j}}=\frac{\tilde{\theta}_{j}}{\tilde{y}_{i}} \bar{S}_{i j}
$$

where $\tilde{y}_{i}$ and $\tilde{\theta}_{j}$ are nominal values of the $i$ th output and $j$ th parameter, respectively, and $\bar{S}_{i j}$ is the steady-state value of the sensitivity coefficient defined in (2). The sensitivity coefficient is nondimensionalized to ensure that contributions of certain parameters and/or outputs are not overemphasized. The sensitivity
TABLE III

DIMENSIONLESS SENSITIVITY MATRIX

\begin{tabular}{c|c|c|c|c}
\hline & $X_{M_{1}}$ & $X_{M_{3}}$ & $P_{r}$ & $\bar{M}_{w}$ \\
\hline$k_{a}$ & -0.07992 & -0.06031 & 0.2287 & -0.4865 \\
\hline$k_{x 3}$ & 0.07983 & 0.06023 & -0.2284 & 0.486 \\
\hline$k_{i 1}$ & $-1.64 \times 10^{-5}$ & $-3.42 \times 10^{-6}$ & $1.85 \times 10^{-4}$ & $-5.93 \times 10^{-4}$ \\
\hline$k_{i 2}$ & $-2.26 \times 10^{-4}$ & $-1.90 \times 10^{-4}$ & $5.08 \times 10^{-4}$ & $-8.83 \times 10^{-4}$ \\
\hline$k_{11}$ & 0.04858 & -0.1014 & 0.05989 & 0.1308 \\
\hline$k_{12}$ & -0.1256 & -0.1588 & 0.1489 & 0.1434 \\
\hline$k_{13}$ & -0.06286 & 0.1487 & 0.1912 & -0.3742 \\
\hline$k_{21}$ & -0.01368 & -0.009504 & 0.05932 & -0.02531 \\
\hline$k_{22}$ & -0.01251 & -0.01139 & 0.01562 & 0.006613 \\
\hline$k_{31}$ & $-1.04 \times 10^{-3}$ & $-8.27 \times 10^{-4}$ & $-2.97 \times 10^{-3}$ & $-7.40 \times 10^{-4}$ \\
\hline$k_{t}$ & 0.08202 & 0.06543 & -0.2345 & 0.3482 \\
\hline$k_{t 2}$ & 0.03064 & 0.02445 & -0.0876 & 0.1300 \\
\hline$k_{t r 1}$ & $3.68 \times 10^{-5}$ & $2.68 \times 10^{-5}$ & $-2.53 \times 10^{-4}$ & -0.5147 \\
\hline$k_{t r_{M 2}}$ & $-2.26 \times 10^{-5}$ & $-2.70 \times 10^{-5}$ & $2.70 \times 10^{-5}$ & -0.07221 \\
\hline
\end{tabular}

coefficient matrix $\bar{S}=\left\{\bar{S}_{i j}\right\}$ can be computed by solving the following steady-state sensitivity equations [3]:

$$
\begin{aligned}
& 0=f(\bar{x}, \bar{u}, \theta) \\
& 0=\frac{\partial f(\bar{x}, \bar{u}, \theta)}{\partial \theta}+\frac{\partial f(\bar{x}, \bar{u}, \theta)}{\partial x} \frac{\partial \bar{x}}{\partial \theta} \\
& \bar{y}=h(\bar{x}) \\
& \bar{S}=\frac{\partial \bar{y}}{\partial \theta}=\frac{\partial h(\bar{x})}{\partial x} \frac{\partial \bar{x}}{\partial \theta}
\end{aligned}
$$

where $\bar{u}, \bar{x}$, and $\bar{y}$ denote steady-state values.

For complex models the sensitivity equations can be difficult to formulate and solve. In this case, an approximate method where the steady-state model equations are solved numerically for small parameter perturbations may be more convenient. Consider a perturbation in the $j$ th parameter, $\Delta \theta_{j}=\theta_{j}-\tilde{\theta}_{j}$, and the corresponding change in the $i$ th output, $\Delta y_{i}=y_{i}-\tilde{y}_{i}$. The approximate sensitivity coefficient is calculated as

$$
S_{i j}=\frac{\partial y_{i}}{\partial \theta_{j}} \approx \frac{\Delta y_{i}}{\Delta \theta_{j}}
$$

In addition to the approximate nature of (11), the need to specify the magnitude of the parameter perturbation introduces some degree of arbitrariness. We have applied both sensitivity-calculation methods to the EPDM reactor model. The two methods yielded virtually identical sensitivity values when perturbations of less than one percent of the nominal parameter values were used. Consequently, only the results obtained by explicitly solving the sensitivity equations are presented in the paper.

Table III shows the dimensionless sensitivity matrix $\tilde{S}$ calculated using the nominal parameter values in Table II. Only the fourteen nonzero parameters are considered. Parameters such as the initiation rate constants $\left(k_{i 1}, k_{i 2}\right)$ and the diene-ethylene rate constant $\left(k_{31}\right)$ have very little effect on the four outputs. They effectively are unidentifiable and should be eliminated from consideration as estimated parameters. However, we include these parameters in the subsequent analysis to better illustrate the proposed method. Certain subsets of parameters (e.g., $k_{a}, k_{x 3}$ ) have nearly linearly dependent effects on the output 
TABLE IV

Overall Parameter EfFects

\begin{tabular}{c|c|c|c|c|c}
\hline Parameter & $E$ & Rank & Parameter & $E$ & Rank \\
\hline$k_{a}$ & 0.4457 & 1 & $k_{11}$ & 0.1256 & 8 \\
\hline$k_{x 3}$ & 0.4452 & 2 & $k_{t r}{ }_{2}$ & 0.0607 & 9 \\
\hline$k_{t r 1}$ & 0.4353 & 3 & $k_{21}$ & 0.0511 & 10 \\
\hline$k_{13}$ & 0.3625 & 4 & $k_{22}$ & $7.71 \times 10^{-3}$ & 11 \\
\hline$k_{t}$ & 0.3590 & 5 & $k_{31}$ & $2.30 \times 10^{-3}$ & 12 \\
\hline$k_{t 2}$ & 0.1341 & 6 & $k_{i 2}$ & $8.82 \times 10^{-4}$ & 13 \\
\hline$k_{12}$ & 0.1305 & 7 & $k_{i 1}$ & $5.03 \times 10^{-4}$ & 14 \\
\hline
\end{tabular}

variables. Consequently, the single parameter from each subset which should be selected for estimation must be determined.

A quantitative measure of the overall effect of each parameter on the measured outputs is derived by applying principal component analysis (PCA) [13] to the dimensionless-sensitivity matrix. The principal components are the eigenvectors of the covariance matrix $X=\tilde{S}^{T} \tilde{S}$. The first principal component is the eigenvector associated with the largest eigenvalue of the $X$-matrix and represents the direction of largest overall variation. The remaining principal components are ordered according to their contribution to the overall variance. $X$ is a $p \times p$ matrix where $p$ is the number of nonzero parameters. Given $m<p$ independent outputs, only $m$ of the eigenvalues of $X$ are nonzero since $\tilde{S}$ is a $m \times p$ matrix. In the EPDM reactor problem $p=14$ and $m=4$. The absolute value of the principal components element $C_{i j}$ reflects the contribution of the $j$ th parameter to the variance of the $i$ th output. The weighted sum of the nonzero eigenvalues $\lambda_{i}$ and their corresponding principal component elements is used as a measure of the overall effect of the $j$ th parameter

$$
E_{j}=\frac{\sum_{i=1}^{m}\left|\lambda_{i} C_{i j}\right|}{\sum_{i=1}^{m}\left|\lambda_{i}\right|}
$$

where $0 \leq E_{j} \leq 1$. The measure $E_{j}$ reflects the difficulty in determining the $j$ th parameter when only a single parameter is estimated. Accurate estimation from limited data sets is favored by large $E_{j}$ values. However, this measure does not account for the quantity and quality of the available data.

Table IV shows the results of the PCA-based calculation (12) for the EPDM reactor model. Based on the relative values of the measure, a preliminary partitioning of parameters into three groups can be performed. Parameters in the first group $\left(k_{a}, k_{x 3}\right.$, $k_{t r 1}, k_{13}, k_{t}$ ) have a comparatively large effect on the four outputs. Accurate estimation of each parameter is precluded only by the presence of linear dependencies with other parameters in this group. Parameters in the second group $\left(k_{t 2}, k_{12}, k_{11}\right.$, $k_{t r_{M 2}}, k_{21}$ ) have a much weaker effect on the outputs. There is the potential to estimate each parameter from this group which has a linearly independent effect. Parameters in the third group $\left(k_{22}, k_{31}, k_{i 2}, k_{i 1}\right)$ have very little effect and cannot be accurately estimated. As discussed below, a more definitive ranking requires investigation of possible linear dependencies between individual parameters as well as the development of a formal procedure to achieve an acceptable tradeoff between the magnitude and linear independence of parameter effects.

\section{B. Linear Dependence of Parameter Effects}

In addition to having a large overall effect, each estimated parameter should have a distinct effect on the measured outputs.
More precisely, the effect of a given parameter should not be linearly dependent with the effects of other estimated parameters. Otherwise, the individual parameter effects cannot be distinguished from the given outputs. Such linear dependencies can be identified from the dimensionless sensitivity matrix $\tilde{S}$. If the sensitivity vector of a given parameter is close to being linearly dependent with the sensitivity vectors of other estimated parameters, then estimation of the given parameter from limited plant data will be problematic. Instead of generating unique solutions over a range of initial parameter values, the estimator (5) will tend to converge to different estimates of the nearly linearly dependent parameters according to the initial value chosen. For example, estimation of both rate constants $k_{a}$ and $k_{x 3}$ in the EPDM problem is expected to be problematic because their effects are nearly linearly dependent (see Table III).

Successful estimation from limited parameter data requires that the sensitivity vectors of the estimated parameters are not too close to being linearly dependent. To formalize this concept, a metric is introduced for the degree of linear independence between the sensitivity vector of a particular parameter and the sensitivity vectors of the other estimated parameters. Let $\tilde{s}_{k}$ represent the dimensionless-sensitivity vector of the $k$ th parameter where $k \in[1, n]$ and $n$ is strictly less than the number of measurements $(m)$. Assuming the $\tilde{s}_{k}$ are linearly independent, any vector in the $n$-dimensional vector space $\tilde{S}_{n}$ can be represented as

$$
\tilde{s}=\sum_{k=1}^{n} a_{k} \tilde{s}_{k}
$$

where the $a_{k}$ are constants. Consider another parameter $j$ with dimensionless-sensitivity vector $\tilde{s}_{j}$ where $j>n$. The objective is to find the vector $\tilde{s} \in \tilde{S}_{n}$ closest to the sensitivity vector $\tilde{s}_{j}$ by solving the following:

$$
\min _{a_{k}} \frac{1}{2}\left(\tilde{s}_{j}-\tilde{s}\right)^{T}\left(\tilde{s}_{j}-\tilde{s}\right) .
$$

This minimization problem is equivalent to solving the following matrix:

$$
\left[\begin{array}{ccc}
\tilde{s}_{1}^{T} \tilde{s}_{1} & \cdots & \tilde{s}_{n}^{T} \tilde{s}_{1} \\
\vdots & \ddots & \vdots \\
\tilde{s}_{1}^{T} \tilde{s}_{n} & \cdots & \tilde{s}_{n}^{T} \tilde{s}_{n}
\end{array}\right] a=\left[\begin{array}{c}
\tilde{s}_{j}^{T} \tilde{s}_{1} \\
\vdots \\
\tilde{s}_{j}^{T} \tilde{s}_{n}
\end{array}\right] .
$$

Given the linear independence of the $\tilde{s}_{k}$, this matrix always is invertible and (15) can be solved for the constant vector $a$.

Next, a measure for the degree of linear independence between the given sensitivity vector $\tilde{s}_{j}$ and the minimum-distance sensitivity vector $\tilde{s}$ derived from (13) is introduced. We have found that the sine of the angle between the sensitivity vectors is a reasonable metric

$$
d_{j}=\sin \left[\cos ^{-1}\left(\frac{\tilde{s}_{j}^{T} \tilde{s}}{\left\|\tilde{s}_{j}\right\|\|\tilde{s}\|}\right)\right]
$$

where $\|\cdot\|$ denotes the Euclidean norm and $d_{j} \in[0,1]$. Two limiting cases are readily identified. When $d_{j}=1$ the sensitivity vector $\tilde{s}_{j}$ is orthogonal to the vector space spanned by the other sensitivity vectors $\tilde{s}_{k}$. Then the effect of $j$ th parameter on the outputs is readily distinguished from the effects of the other parameters. Conversely, $\tilde{s}_{j}$ is linearly dependent with one or more 
of the sensitivity vectors $\tilde{s}_{k}$ when $d_{j}=0$. In this case, the individual effect of the $j$ th parameter cannot be distinguished from the collective effect of the other parameters regardless of the amount of steady-state plant data. A potential shortcoming of the proposed method is that parameter effects which are indistinguishable from steady-state information may be distinguishable from dynamic data. Parameters which yield comparatively large values of the linear-independence metric $d_{j}$ are preferred for estimation.

We have used the metric (16) to identify the possible presence of nearly linearly dependent parameters in the EPDM-reactor model given the nominal parameter values in Table II. While not shown here for the sake of brevity, the results demonstrate that the quadruplet $\left\{k_{a}, k_{x 3}, k_{t}, k_{t_{2}}\right\}$ and the pair $\left\{k_{t r 1}, k_{t r_{M 2}}\right\}$ form nearly linearly dependent parameter groups. This suggests that only one parameter from each group can be successfully estimated from limited plant data. A more formal analysis procedure based on this metric is introduced below.

\section{Parameter Selection Procedure}

We propose a method for selecting estimated parameters based on a quantitative measure of practical identifiability. The identifiability measure provides a tradeoff between the two objectives discussed above: i) large effect of each parameter on the output variables as measured by the magnitude $E_{j}$ and ii) linear independence of the parameter effects on the output variables as measured by the metric $d_{j}$. The following recursive algorithm is used to rank each candidate parameter according to its identifiability.

1) Perform the PCA-based calculation (12) to determine the overall effect $E_{j}$ of each parameter $\theta_{j}$. Select the highest ranked parameter $p_{1}=\left\{\theta_{k} \mid E_{k}=\max _{j} E_{j}\right\}$ and set the number of selected parameters $n=1$.

2) Let $m$ represent the number of measured outputs. If $n<m$, then use (16) to compute the linear-independence metric $d_{j}$ for each remaining parameter $\theta_{j}$ with respect to previously selected parameters $\left\{p_{1}, \ldots, p_{n}\right\}$. Go to step 4 .

3) If $n \geq m$, then form all possible $(m-1)$-tuples of the previously selected parameters. The number of possible combinations is $q=(n ! /((m-1) !(n-m+1) !))$. Use (16) to compute the linear-independence metric $d_{q, j}$ for each remaining parameter $\theta_{j}$ with respect to every combination of the previously selected parameters $\left\{p_{1}, \ldots, p_{m-1}\right\}$. Determine the worse-case metric over all the combinations: $d_{j}=\min _{q} d_{q, j}$.

4) Calculate the identifiability index $I_{j}$ for each remaining parameter $\theta_{j}$

$$
I_{j}=E_{j} d_{j}
$$

Select the next highest ranked parameter $p_{n+1}=$ $\left\{\theta_{k} \mid I_{k}=\max _{j} I_{j}\right\}$, and set the number of selected parameters $n=n+1$. If $n<p$ where $p$ is the number of parameters, then return to step 2. Otherwise, terminate the algorithm.

The only consideration involved in choosing the first parameter is its overall effect on the measured outputs. Remaining pa-
TABLE V

Results of Two PARAMETER SELECTION ALgORITHMS

\begin{tabular}{c|l|l}
\hline $\mathrm{n}$ & Proposed Method & FIM-Based Method \\
\hline 1 & $k_{a}$ & $k_{12}$ \\
\hline 2 & $k_{a}, k_{t r 1}$ & $k_{12}, k_{13}$ \\
\hline 3 & $k_{a}, k_{t r 1}, k_{13}$ & $k_{12}, k_{13}, k_{t r 1}$ \\
\hline 4 & $k_{a}, k_{t r 1}, k_{13}, k_{11}$ & $k_{12}, k_{13}, k_{t r 1}, k_{11}$ \\
\hline 5 & $k_{a}, k_{t r 1}, k_{13}, k_{11}, k_{12}$ & $k_{12}, k_{13}, k_{t r 1}, k_{11}, k_{a}$ \\
\hline 6 & $k_{a}, k_{t r 1}, k_{13}, k_{11}, k_{12}, k_{t}$ & $k_{12}, k_{13}, k_{t r 1}, k_{11}, k_{a}, k_{t 2}$ \\
\hline 7 & $k_{a}, k_{t r 1}, k_{13}, k_{11}, k_{12}, k_{t}, k_{21}$ & $k_{12}, k_{13}, k_{t r 1}, k_{11}, k_{x 3}, k_{t 2}, k_{21}$ \\
\hline
\end{tabular}

rameters are ranked to provide an acceptable tradeoff between the magnitude and linear independence of their effects as measured by the identifiability index $I_{j}$. The linear-independence metric $d_{j}$ must be computed subject to the constraint that the vector space spanned by the sensitivity vectors of the previously selected parameters has maximum dimension $m-1$ where $m$ is the number of measured outputs. Otherwise, the sensitivity vector of any remaining parameter is necessary contained in this vector space since the sensitivity vector has rank $m$. When the number of previously selected parameters $(n)$ is strictly less than $m$, the vector space considered is spanned by the sensitivity vectors of all previously selected parameters. When $n \geq m$, then $q$ different vector spaces are constructed from all possible $(m-1)$-tuples of the sensitivity vectors of the previously selected parameters. The linear-independence metricis computed with respect to the worse-case vector space in the sense that the metric $d_{q, j}$ is minimized over all possible combinations of the previously selected parameters. The final result of the procedure is a ranking of the candidate parameters according to their identifiability.

A potential shortcoming of the proposed method is that the total number $q$ of $(m-1)$-tuples grows rapidly as the number of parameters $p$ and the number of measurements $m$ increase. For the EPDM reactor example where $p=14$ and $m=4$, the maximum number of combinations which must be evaluated during a single iteration is 364 . The selection procedure can become computationally prohibitive for larger $p$ and $m$. Although not discussed here, a simpler linear-dependence test based on collinearity between parameter pairs can be used to substantially reduce the computational effort at expense of neglecting linear dependencies between three of more parameters [14].

Results for the first seven iterations of the proposed algorithm for the EPDM-reactor model are shown in the second column of Table V. The rate constant $k_{a}$ is ranked first because it has the largest overall effect on the measured outputs (see Table IV). Despite being the second highest ranked parameter with respect to overall effect, $k_{x 3}$ is not chosen in the first seven steps because its sensitivity vector is nearly linearly dependent with that of $k_{a}$. As a result, $k_{t r 1}$ and $k_{13}$ are ranked second and third, respectively. The parameter $k_{t}$ is not selected until the sixth iteration because its sensitivity vector is nearly linearly dependent with that of $k_{a}$. The parameter $k_{t 2}$ is not chosen in the first seven steps for the same reason. Despite having a slightly smaller overall effect, the parameter $k_{11}$ is ranked just above $k_{12}$ because $k_{11}$ has a larger linear-independence metric. The parameter $k_{21}$ is ranked seventh because the sensitivity vector of $k_{t r_{M 2}}$ is nearly linearly dependent with that of $k_{t r 1}$. 
As originally proposed, the FIM-based method [12] discussed in Section I requires a subjective choice of the parameters that yield the best tradeoff between minimization of the FIM-condition number $(C)$ and minimization of the FIM determinant $(D)$. To eliminate this subjectivity, we have modified the FIM-based approach such that the estimated parameters are chosen to minimize the product of these two measures. This ensures that a unique solution is obtained for each subset of candidate parameters. The third column of Table $\mathrm{V}$ shows the results of the first seven iterations of the modified FIM-based algorithm. The proposed and FIM-based methods yield different parameter sets at each iteration. For instance, $k_{12}$ is selected first in the FIM-based algorithm despite being only the seventh ranked parameter in terms of overall effect (see Table IV). Furthermore, the FIM-based algorithm does not simply add another parameter to the previously selected parameters. For example, in the seventh iteration of the FIM algorithm the parameter $k_{a}$ selected in the fifth iteration is dropped while $k_{x 3}$ and $k_{21}$ are added. The implications of these differences are examined in the next section by performing off-line parameter-estimation tests.

\section{OFF-Line PARAMETER-ESTIMATION RESUlts}

While a ranking of parameters according to their identifiability is generated, the proposed method does not directly address the question of the number of parameters that should be estimated. We have found that differences between identifiability measures can provide some useful information about the number of estimated parameters. If the identifiability measure of the $N$ th ranked parameter is much greater than that of the $(N+1)$ th ranked parameter, then estimation of the $(N+1)$ th parameter likely will be difficult. However, this heuristic is not generally applicable because some problems will not admit such a clear distinction. As an alternative, we perform limited off-line parameter-estimation tests to determine the appropriate number of estimated parameters. While this section focuses on off-line estimation, the parameter selection technique is equally applicable to on-line estimation where the number of estimated parameters must be equal to the number of measurements to ensure observability of the augmented system [27], [28].

\section{A. Simulated Plant Data}

Simulated plant data is obtained from a proprietary EPDMreactor model that utilizes a different kinetic scheme than that shown in Table I to model polymer-chain growth. This is referred to as the plant model in the subsequent simulation studies. The dynamic model based on the kinetic scheme in Table I that is used for solution of the off-line estimation problem is referred to as the estimation model. Due to differences in the kinetic schemes, there are structural-modeling errors between the plant and estimation models even if the same kinetic-rate constants are used.

The plant model is used to generate dynamic data for parameter estimation. The feed-molar flow rates of the various reactants are available for plant excitation. Because optimal experimental design is outside the scope of this paper, a process knowledge based approach is used to design the plant-input sequence
TABLE VI

Plant INPUT SEQUENCE

\begin{tabular}{c|c|c|c|c|c}
\hline Feed & $M_{1}$ & $M_{2}$ & $M_{3}$ & $C_{1}, A l$ & $H_{2}$ \\
\hline$-25 \%$ step change & $0.5 \mathrm{~h}$ & $2 \mathrm{~h}$ & $3.5 \mathrm{~h}$ & $5 \mathrm{~h}$ & $6.5 \mathrm{~h}$ \\
\hline$+50 \%$ step change & $1 \mathrm{~h}$ & $2.5 \mathrm{~h}$ & $4 \mathrm{~h}$ & $5.5 \mathrm{~h}$ & $7 \mathrm{~h}$ \\
\hline$-25 \%$ step change & $1.5 \mathrm{~h}$ & $3 \mathrm{~h}$ & $4.5 \mathrm{~h}$ & $6 \mathrm{~h}$ & $7.5 \mathrm{~h}$ \\
\hline
\end{tabular}

TABLE VII

COMPARISON OF Two PARAMETER SELECTION METHOdS

\begin{tabular}{c|c|c|c|c|c|c}
\hline \multirow{3}{*}{$\begin{array}{c}\text { Proposed } \\
\text { Method }\end{array}$} & & $k_{a}$ & $k_{11}$ & $k_{13}$ & $k_{t r 1}$ & $\mathrm{~J}$ \\
\cline { 2 - 7 } & Initial value & 0.0366 & 0.0542 & 0.5842 & 2.2236 & \\
\cline { 2 - 7 } & Solution & 0.7175 & 0.3796 & 0.3957 & 1.6655 & 0.0017 \\
\cline { 2 - 7 } & Initial value & 3.1604 & 0.0877 & 0.3925 & 0.2290 & \\
\cline { 2 - 7 } & Solution & 0.6663 & 0.4126 & 0.4205 & 1.7215 & 0.0017 \\
\hline \multirow{3}{*}{$\begin{array}{c}\text { FIM-Based } \\
\text { Method }\end{array}$} & & $k_{11}$ & $k_{12}$ & $k_{13}$ & $k_{t r 1}$ & $\mathrm{~J}$ \\
\cline { 2 - 7 } & Initial value & 0.0366 & 0.0542 & 0.5842 & 2.2236 & \\
\cline { 2 - 7 } & Solution & 0.5322 & 0.8841 & 0.5447 & 2.4311 & 0.0031 \\
\cline { 2 - 7 } & Initial value & 3.1604 & 0.0877 & 0.3925 & 0.2290 & \\
\cline { 2 - 7 } & Solution & 0.5353 & 0.8853 & 0.5454 & 2.4326 & 0.0031 \\
\hline
\end{tabular}

shown in Table VI. The sequence consists of three step changes in each feed flow rate of the magnitude and duration listed. The ratio of the catalyst and cocatalyst flow rates is unchanged because these flow rates must be maintained at a certain stoichiometric ratio. Actual flow rates cannot be revealed for proprietary reasons.

\section{B. Comparison of Parameter Selection Methods}

The proposed parameter-selection method is compared to the FIM-based technique described in Section III-C to determine potential advantages associated with these alternative approaches. As shown in Table VII, for the case of four estimated parameters $(n=4)$, the rate constants $k_{11}, k_{13}$, and $k_{t r 1}$ are selected by both methods. However, the fourth parameter chosen is $k_{a}$ with the proposed method and $k_{12}$ with the FIM-based method. The two sets of selected parameters are compared by solving the off-line estimation problem (5) using the simulated plant data discussed in Section IV-A. Table VII shows the results obtained for two different sets of initial parameter values that were obtained by randomly perturbing the nominal values in Table II. For both initial conditions, each method converges to a small region in the parameter space. Because the two optimization problems differ only with respect to the decision variables, the objective-function value provides a direct measure of the relative performance that can be achieved with a given set of estimated parameters. The proposed method yields an objective-function value that is approximately half the value obtained with the FIM-based technique. When viewed in conjunction with the previously discussed shortcomings of FIM-based methods, including the heuristic nature of the parameter selection process and computationally inefficiency, these result suggest that the proposed method is potentially superior for problems with a large number of unknown parameters. However, this conclusion is based on the results of a single case study. Several additional studies would be required to determine if the proposed method is generally superior or if the preferred method is application dependent. 
TABLE VIII

Five PARAMETER Estimation Results FOR PeRFeCt MODEL

\begin{tabular}{c|c|c|c|c|c|c|c}
\hline & $k_{a}$ & $k_{11}$ & $k_{12}$ & $k_{13}$ & $k_{t r 1}$ & $J$ & SSE \\
\hline Plant value & 0.3663 & 0.4212 & 0.6886 & 0.4139 & 1.7582 & & \\
\hline Initial value & 0.0366 & 0.0542 & 0.5842 & 2.2236 & 3.1604 & & \\
\hline Solution & 0.3710 & 0.4062 & 0.6964 & 0.4104 & 1.7355 & 0.000591 & 0.0018 \\
\hline Initial value & 0.0877 & 0.3925 & 0.2290 & 0.1229 & 1.1242 & & \\
\hline Solution & 0.3581 & 0.4288 & 0.6996 & 0.4200 & 1.7875 & 0.00041 & 0.0016 \\
\hline
\end{tabular}
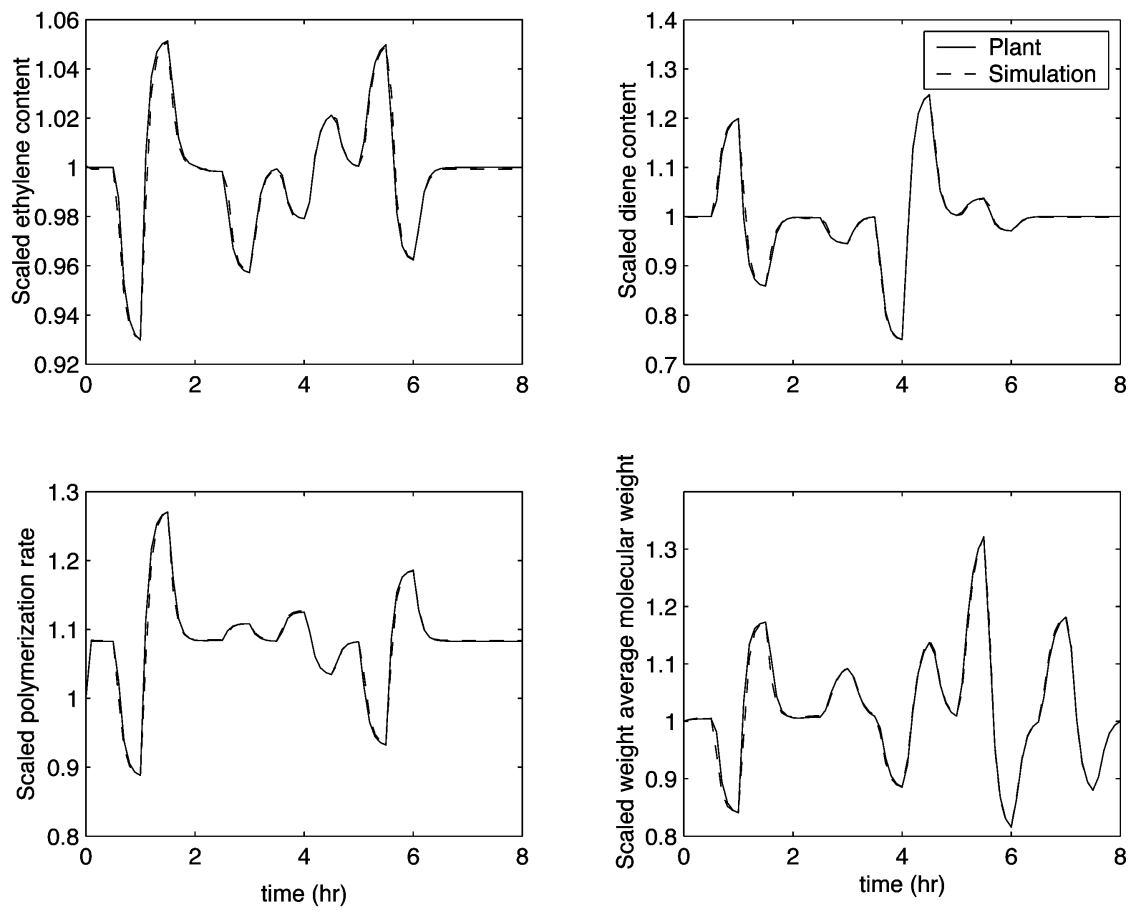

Fig. 1. Comparison of dynamic responses for perfect model with five estimated parameters.

\section{Determination of the Number of Estimated Parameters}

We utilize off-line parameter-estimation tests to determine the appropriate number of estimated parameters for the EPDM problem. The objective is to estimate as many parameters as possible subject to the restriction that the optimal solution is not too strongly dependent on the initial parameter values. We refer to this property as reliable parameter estimation. Four estimated parameters is viewed as a minimum number because the dimensionless sensitivity matrix has rank four at the nominal steady state. If the reliability property does not hold, then the final parameter values obtained are arbitrarily determined by the initial parameter values chosen. Given this situation, the preferred alternative is to reduce the number of estimated parameters such that unique estimates are obtained from different initial values. Below we present two sets of simulation tests designed to analyze estimation performance. The first set of tests are designed to analyze parameter convergence in the absence of structural modeling errors, while the second set of tests are designed to investigate the effects of plant/model mismatch.

In the first set of tests, the estimation model is used as the plant model to eliminate structural modeling errors. The initial values of the estimated parameters are obtained by random perturbation of their nominal values in Table II while the nonestimated parameter values are equal to their nominal values.
Table VIII shows results obtained by solving the off-line estimation problem (5) where the first five parameters in the second column of Table $\mathrm{V}$ are used as decision variables. The dynamic plant data is obtained by using the input sequence in Table VI to perturb the estimation model. For each initial condition considered, the five estimated parameters converge to a small region that contains the plant values and the sum of squared errors (SSE) between the estimated and plant parameter values is small. The small differences between the two optimal solutions are attributable to the random nature of the simulated-annealing algorithm. Predicted dynamic responses of the scaled output variables obtained using the first set of five estimated parameters in Table VIII are compared to the plant responses in Fig. 1. Only very small differences between the model and plant responses are observed. The results suggest that these five parameters can be reliably estimated from the available plant data.

Table IX shows estimation results when the first six parameters in the second column of Table $\mathrm{V}$ are used as decision variables in the optimization problem. The estimates do not converge to a region near the plant values as demonstrated by the large SSE value. The lack of parameter convergence is attributable to the inclusion of $k_{t}$, whose effect is nearly linearly dependent with that of $k_{a}$. Fig. 2 shows a comparison of the plant responses and the model responses obtained with the six estimated parameters. As compared to the five parameter case 
TABLE IX

SiX Parameter Estimation Results FOR PERFECT MODEL

\begin{tabular}{c|c|c|c|c|c|c|c|c}
\hline & $k_{a}$ & $k_{11}$ & $k_{12}$ & $k_{13}$ & $k_{t}$ & $k_{t r 1}$ & $\mathrm{~J}$ & SSE \\
\hline Plant value & 0.3663 & 0.4212 & 0.6886 & 0.4139 & 0.5604 & 1.7582 & & \\
\hline Initial value & 0.0366 & 0.0542 & 0.5842 & 2.2236 & 3.1604 & 0.0877 & & \\
\hline Solution & 0.4193 & 2.0351 & 3.4016 & 2.0522 & 3.0929 & 5.5253 & 0.002675 & 70.9 \\
\hline
\end{tabular}
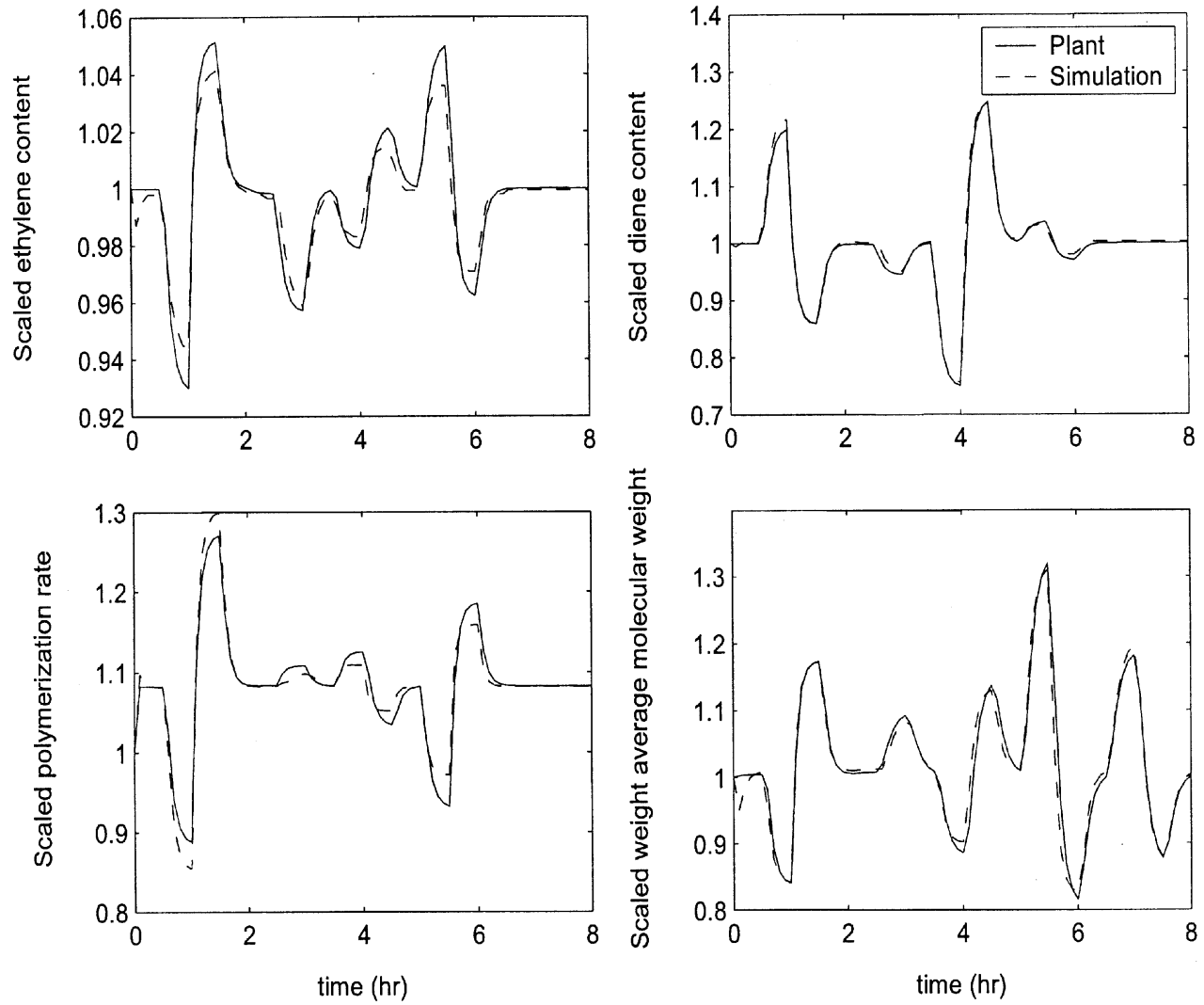

Fig. 2. Comparison of dynamic responses for perfect model with six estimated parameters.

shown in Fig. 1, predictions of the polymerization rate and weight-average molecular weight are much less accurate. These results suggest that reliable estimation of six kinetic parameters is possible only with the design of a more exciting input sequence and/or the inclusion of additional measurements. Both strategies can be problematic in a EPDM-plant environment.

In the second set of tests, a proprietary reactor model is used as the simulated plant to investigate the effects of structural modeling errors. The initial parameter values used in the optimization problem are obtained by randomly perturbing the nominal values in Table II. Due to plant/model mismatch, the estimated parameter values cannot be expected to converge to the plant values. Consequently, the estimation results are analyzed with respect to convergence from different initial parameter values, the objective function values and the predicted dynamic responses. Table $\mathrm{X}$ shows estimation results obtained using the first five parameters in the second column of Table V. The estimates converge to the same small region of parameter space for both sets of initial values. Fig. 3 shows dynamic simulation results obtained using the estimated parameters derived from the first set of initial values. Although some disparities between the plant and model responses are evident, the predictions
TABLE $X$

Five PARAMETER ESTIMATION RESULTS FOR IMPERFECT MODEL

\begin{tabular}{c|c|c|c|c|c|c}
\hline & $k_{a}$ & $k_{11}$ & $k_{12}$ & $k_{13}$ & $k_{t r 1}$ & $\mathrm{~J}$ \\
\hline Initial value & 0.0366 & 0.0542 & 0.5842 & 2.2236 & 3.1604 & \\
\hline Solution & 1.2442 & 0.3544 & 0.5760 & 0.3473 & 1.3205 & 0.001458 \\
\hline Initial value & 0.0877 & 0.3925 & 0.2290 & 0.1229 & 1.1242 & \\
\hline Solution & 1.5027 & 0.3385 & 0.5536 & 0.3275 & 1.2447 & 0.001533 \\
\hline
\end{tabular}

are acceptable despite the presence of structural modeling errors. Although not shown here, the estimation results obtained using the first six parameters in the second column of Table $\mathrm{V}$ exhibit poor parameter convergence, large objective function values, and biased output predictions. These results reinforce the conclusion that only five kinetic parameters can be reliably estimated given the available measurements and dynamic data.

\section{SUMMARY AND CONCLUSION}

A simple method for analyzing mechanistic-dynamic models to determine the most appropriate parameters for off-line estimation has been proposed. The steady-state analysis is based on a dimensionless parameter-output sensitivity matrix which is readily derived from the available model. The overall effect 

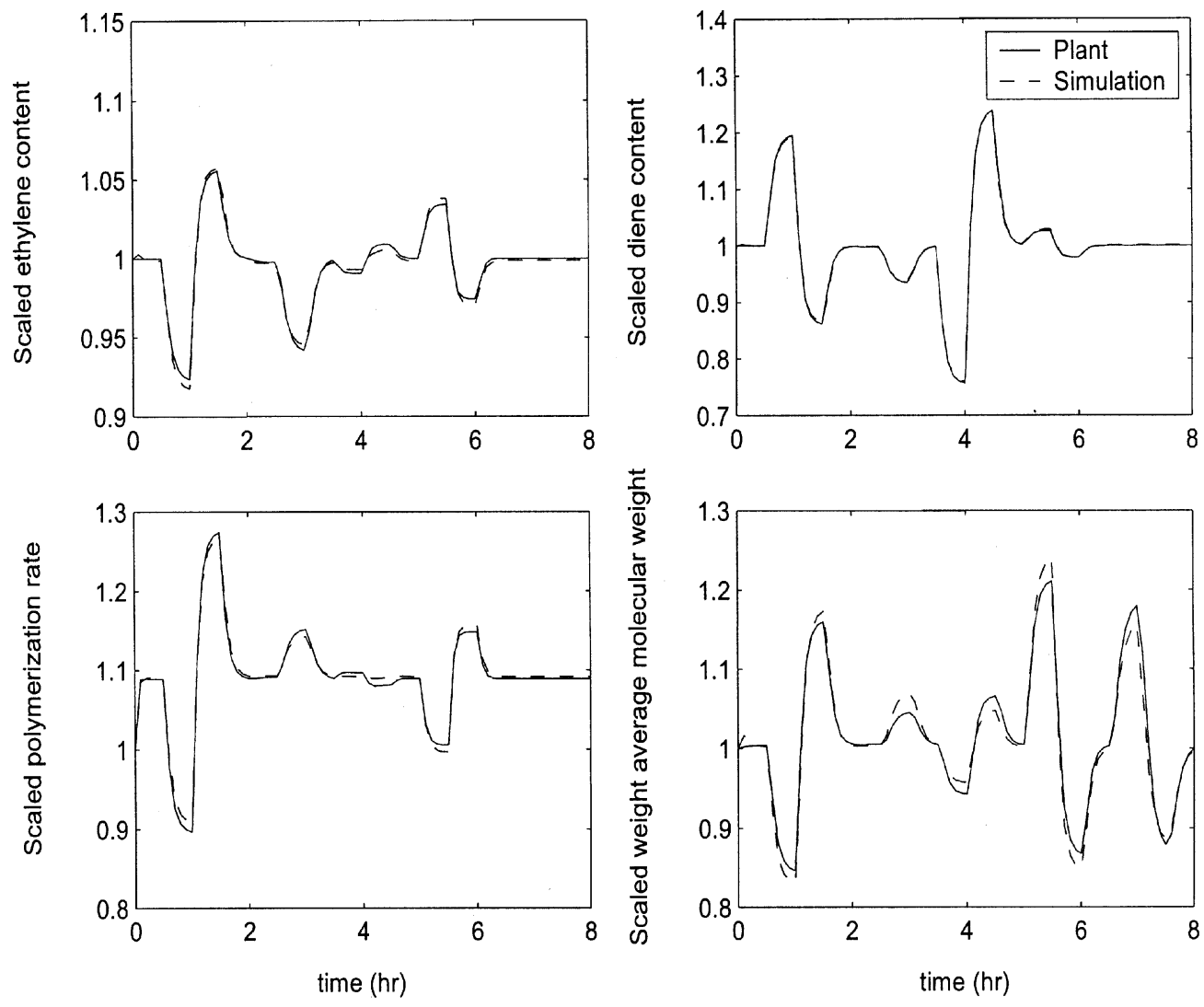

Fig. 3. Comparison of dynamic responses for imperfect model with five estimated parameters.

of each parameter on the measured outputs is determined by applying principal component analysis to the sensitivity matrix. The uniqueness of each parameter effect is determined by computing the minimum distance between the sensitivity vector of the particular parameter and the vector spaces spanned by sensitivity vectors of the parameters already selected for estimation. A recursive parameter-ranking algorithm is used to achieve an acceptable tradeoff between the magnitude and linear independence of parameter effects. The proposed method is successfully applied to the problem of kinetic parameter estimation for an industrial model of a EPDM reactor. Off-line estimation tests suggest that five kinetic parameters can be reliably estimated from four product-quality measurements given the available dynamic-simulation data.

The proposed parameter-selection method is based on the parameter-output sensitivity matrix computed at a particular steady-state operating point. Consequently, the proposed method does not account for dynamic or nonlinear characteristics that may effect parameter identifiability. A dynamic extension is possible if the calculations are performed using a dynamically weighted sensitivity matrix

$$
\tilde{S}_{D}=\sum_{k=1}^{M} w(k) \tilde{S}(k)
$$

where $M$ and $\tilde{S}(k)$ are defined as before and $w(k)$ is a dynamic weighting factor that is used to obtain an appropriate relative weighting of dynamic and steady-state data. The proposed method possibly can be extended to nonlinear steady-state anal- ysis by averaging the sensitivity matrices generated at multiple steady states

$$
\tilde{S}_{N}=\frac{1}{N} \sum_{i=1}^{N} \tilde{S}_{i}
$$

where $N$ is the number of steady-state points and $\tilde{S}_{i}$ is the dimensionless sensitivity matrix at steady-state point $i$. Both of these extensions currently are being studied for the EPDM kinetic parameter-estimation problem.

\section{REFERENCES}

[1] L. Ljung, System Identification: Theory for the User. Englewood Cliffs, NJ: Prentice-Hall, 1999.

[2] T. Söderström and P. Stoica, System Identification. Englewood Cliffs, NJ: Prentice-Hall, 1989.

[3] P. Englezos and N. Kalogerakis, Applied Parameter Estimation for Chemical Engineers. New York: Marcel Dekker, 2001.

[4] A. Gelb, Applied Optimal Estimation. Cambridge, MA: MIT Press, 1974.

[5] J. A. Jacquez and P. Greif, "Numerical parameter identifiablity and estimability: integrating identifiablity, estimability, and optimal sampling design," Math. Biosci., vol. 76, pp. 201-327, 1985.

[6] K. R. Godfrey and J. J. DiStefano, Identification and System Parameter Estimation. Oxford, U.K.: Pergamon, 1985.

[7] R. N. Mehra, "Optimal inputs for linear system identification," IEEE Trans. Automat. Contr., vol. AC-19, pp. 192-200, June 1974.

[8] J. V. Beck and K. J. Arnold, Parameter Estimation in Engineering and Science. New York: Wiley, 1977.

[9] L. L. Scharf and L. McWhorter, "Geometry of the Cramer-Rao bound," Signal Processing, vol. 31, pp. 301-311, 1993.

[10] R. K. Mehra, "Optimal input signals for parameter identification in dynamic systems-survey and new results," IEEE Trans. Automat. Contr., vol. 19, pp. 753-768, Dec. 1974. 
[11] E. Walter and L. Pronzato, "Qualitative and quantitative experimental design for phenomenological models-a survey," Automatica, vol. 26, pp. 145-213, 1990.

[12] S. R. Weijers and P. A. Vanrolleghem, "A procedure for selecting best identifiable parameters in calibrating activated sludge model no. 1 to full-scale plant data," Water Sci. Technol., vol. 36, pp. 69-79, 1997.

[13] G. H. Dunteman, Principal Components Analysis. Newbury Park, CA: Sage, 1989.

[14] P. Mhaskar, M. A. Hjortso, and M. A. Henson, "Cell population modeling and parameter estimation of continuous cultures of Saccharomyces cerevisiae," Biotech. Prog., vol. 18, pp. 1010-1026, 2002.

[15] G. V. Strate, "Ethylene-propylene elastomers," in Encyclopedia of Polymer Science and Engineering. New York: Wiley, 1986, pp. 522-564.

[16] "Hazardous Air Pollutant Emissions From Process Units in the Elastomer Manufacturing Industry-Basis and Purpose Document for Proposed Standards," Office of Air Quality, Environmental Protection Agency, Tech. Rep. EPA-453/R-95-006a, May 1995.

[17] C. Cozewith, "Transient response of continuous-flow stirred-tank polymerization reactors," AIChE J., vol. 34, pp. 272-282, 1988.

[18] C. Cozewith and G. W. V. Strate, "Ethylene-propylene copolymers reactivity ratios, evaluation, and significance," Macromolecules, vol. 4, pp. 482-489, 1971.

[19] B. A. Ogunnaike, "On-line modeling and predictive control of an industrial terpolymerization reactor," Int. J. Control, vol. 59, pp. 711-729, 1994.

[20] E. S. Meadows and J. B. Rawlings, "Model predictive control," in Nonlinear Process Control, M. A. Henson and D. E. Seborg, Eds. Englewood Cliffs, NJ: Prentice-Hall, 1997, ch. 5, pp. 233-310.

[21] C. A. Floudas, Nonlinear and Mixed-Integer Optimization. Oxford, U.K.: Scientific, 1995.

[22] T. F. Edgar, D. Himmelblau, and L. Lasdon, Optimization for Engineering Systems. New York: McGraw Hill, 2001.

[23] S. S. Rao, Optimization Theory and Applications. New York: Wiley, 1984.

[24] D. E. Goldberg, Genetic Algorithms in Search, Optimization, and Machine Learning. Reading, MA: Addison-Wesley, 1989.

[25] A. Corana, M. Marchesi, C. Martini, and S. Ridella, "Minimizing multimodal functions of continuous variables with the simulated annealing algorithm," ACM Trans. Math. Software, vol. 13, pp. 262-280, 1987.

[26] W. L. Goffe, "SIMANN: a global optimization algorithm using simulated annealing," Stud. Nonlinear Dyn. Econometr., vol. 1, pp. 169-176, 1996.

[27] D. J. Kozub and J. F. MacGregor, "State estimation for semi-batch polymerization reactors," Chem. Eng. Sci., vol. 47, pp. 1047-1062, 1992.

[28] N. L. Ricker and J. H. Lee, "Nonlinear modeling and state estimation for the Tennessee Eastman challenge process," Comput. Chem. Eng., vol. 19, pp. 983-1005, 1995.

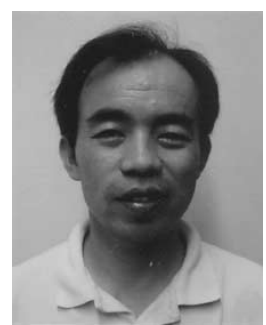

Rujun Li received the B.Sc. and M.Sc. degrees in chemical engineering from Tianjin University, Tianjin, China, in 1992 and 1995, respectively, and the Ph.D. degree in chemical engineering from Louisiana State University, Baton Rouge, LA, in 2003.

From 1995 to 1998, he worked in Peking University Founder Group, Beijing, China, as a Process Engineer and later as the Department Manager. His areas of research are dynamic modeling, parameter estimation, and nonlinear control of chemical

processes.

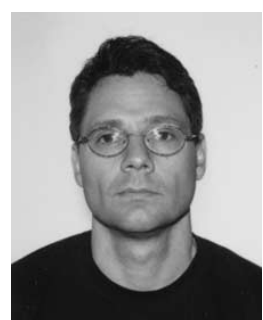

Michael A. Henson received the B.S. degree in chemical engineering from the University of Colorado, Boulder, in 1985, the M.S. degree in chemical engineering from the University of Texas, Austin, in 1988, and the Ph.D. degree in chemical engineering from the University of California, Santa Barbara, in 1992.

He held the position of Visiting Research Scientist with the DuPont Company from 1992 to 1993, the positions of Assistant Professor and Associate Professor of chemical engineering at Louisiana State University from 1994 to 2002, and the position of Alexander von Humboldt Research Fellow at the University of Stuttgart, Germany, from 2001 to 2002 . He currently is an Associate Professor of chemical engineering at the University of Massachusetts, Amherst, MA. He is coeditor of the book Nonlinear Process Control (Englewood Cliffs, NJ: Prentice-Hall, 1997), has authored over 80 book chapters, journal articles and conference proceeding papers and has served as an Associate Editor for the Journal of Process Control since 2000. His current research is focused on nonlinear modeling and control of chemical and biochemical processes.

Dr. Henson received an Early Faculty Career Development Award from the National Science Foundation in 1995.

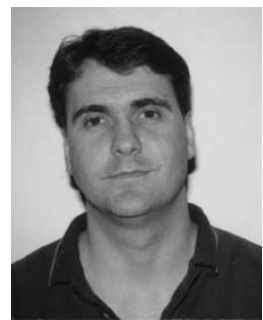

Michael J. Kurtz received the B.S. degree in chemical engineering from Florida State University, Tallahassee, in 1993 and the Ph.D. degree in chemical engineering from Louisiana State University, Baton Rouge, in 1997.

$\mathrm{He}$ is currently the applications engineering section Polymers Group Leader for ExxonMobil Chemical Company, Baton Rouge, LA. His work is in the areas of nonlinear modeling, estimation, and control of polymer processes as well as statistical validation of plant data and control performance monitoring. 